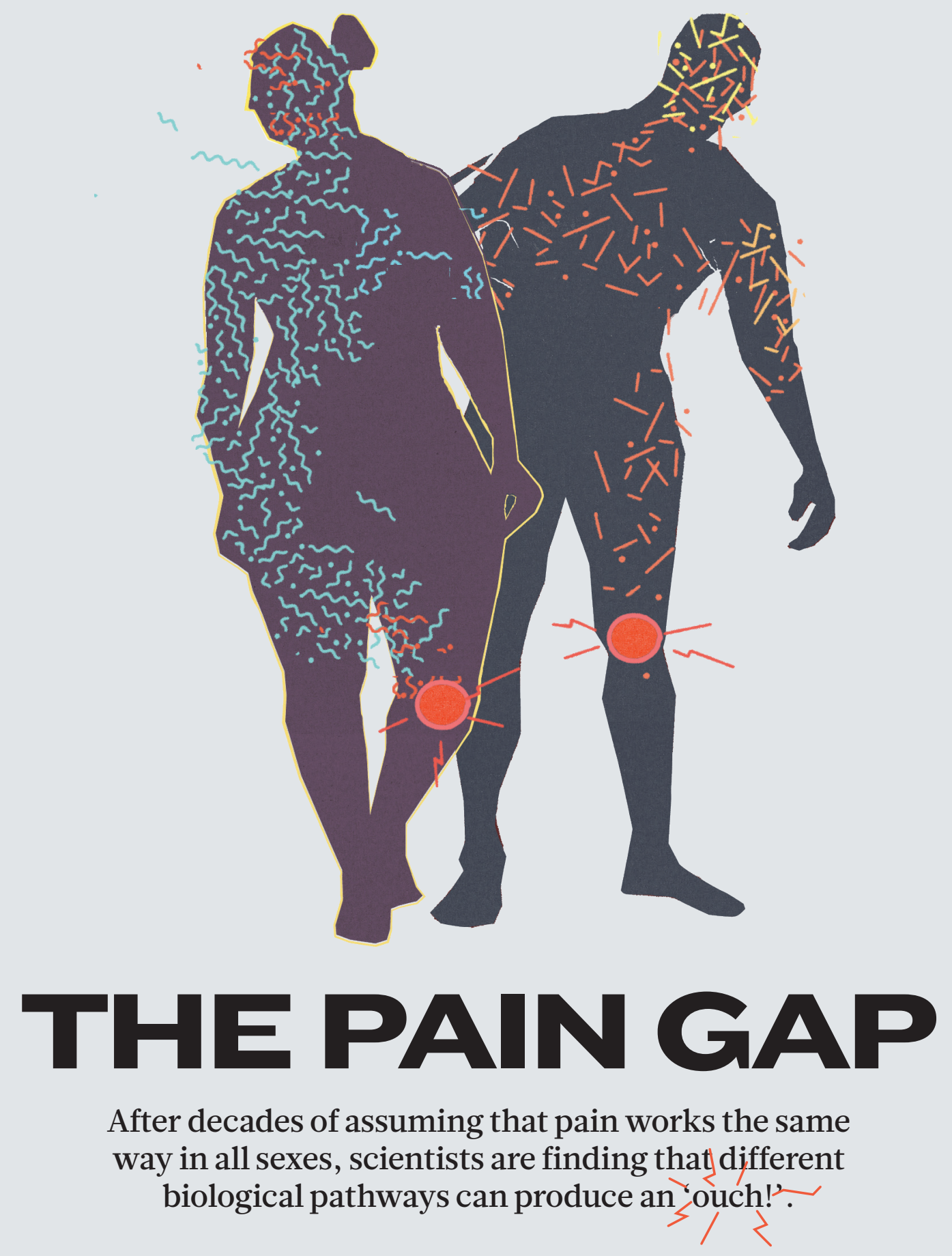

BY AMBER DANCE

$\mathbf{R}$ obert Sorge was studying pain in mice in 2009, but he was the one who ended up with a headache.

At McGill University in Montreal, Canada, Sorge was investigating how animals develop an extreme sensitivity to touch. To test for this response, Sorge poked the paws of mice using fine hairs, ones that wouldn't ordinarily bother them. The males behaved as the scientific literature said they would: they yanked their paws back from even the finest of threads.

But females remained stoic to Sorge's gentle pokes and prods ${ }^{1}$. "It just didn't work in the females," recalls Sorge, now a behaviourist at the University of Alabama at Birmingham. "We couldn't figure out why." Sorge and his adviser at McGill University, pain researcher Jeffrey Mogil, would go on to determine that this kind of pain hypersensitivity results from remarkably different pathways in male and female mice, with distinct immune-cell types contributing to discomfort ${ }^{2}$.

Sorge and Mogil would never have made their discovery if they had followed the conventions of most pain researchers. By including male and female mice, they were going against the crowd. At the time, many pain scientists worried that females' hormone cycles would complicate results. Others stuck with males because, well, that's how things were done.

Today, inspired in part by Sorge and Mogil's work and spurred on by funders, pain researchers are opening their eyes to the spectrum of responses across sexes. Results are starting to trickle out, and it's clear that certain pain pathways vary considerably, with immune cells and hormones having key roles in differing responses.

This push is part of a broader movement 
to consider sex as an important variable in biomedical research, to ensure that studies cover the range of possibilities rather than gleaning results from a single population. A major change came in 2016, when the US National Institutes of Health (NIH) made it a requirement for grant applicants to justify their choice of the sex of animals used in experiments. The discoveries in pain research are among the most exciting to emerge, says Cara Tannenbaum, scientific director of the Institute of Gender and Health in Montreal, part of the Canadian Institutes of Health Research. And of Sorge and Mogil's work, she adds, "To my knowledge, no other field of science has identified this type of sex difference."

The research could open the door for new medical advances, adds Tannenbaum. These are sorely needed: some $20 \%$ of people worldwide experience chronic pain - and the majority are women. Today, the pharmaceutical market offers the same pain drugs to everyone. But if the roots of pain are different, some drugs might work better in some people than in others.

Moreover, people might require different pain medications when hormone levels fluctuate through life. And a person's sex doesn't always fit clearly into the categories of male and female: it is determined by a spectrum of characteristics, including genetics, anatomical development and hormone levels, each of which might affect a person's needs in pain therapy. The picture is a long way from complete, and studies - most in rodents - have so far focused on biological sex, as opposed to gender, a psychosocial concept that doesn't necessarily match sex.

Iain Chessel, vice-president and head of neuroscience at AstraZeneca in Cambridge, UK, predicts that future pain medications will be tailored to individuals - and that sex will be a key factor in those personalized prescriptions. "But we don't understand it yet," he adds.

\section{IMMUNE TO THE PAIN}

Pain happens when neural sensors in the skin, muscles, joints or organs register a potentially harmful sensation, such as heat or tissue damage. They send signals through peripheral nerves to the spinal cord, activating other nerves that send signals to the brainstem and on to the cerebral cortex, which interprets those signals as 'ouch!'. But pain happens in many ways, and diverse chemical pathways contribute. Some pain types are distinguished by timing. There's the acute response to something hot, sharp or otherwise noxious, and there's long-term, chronic pain that might persist even after the initial injury has healed.

Chronic pain can manifest as hypersensitivity to otherwise non-painful stimuli, as in the case of Sorge's male mice. Back in 2009, he and Mogil were studying a model of chronic pain triggered by inflammation.

Injecting a bacterial molecule called lipopolysaccharide into the spines of mice drew the attention of microglia, the nervous system's resident immune cells. But in Sorge's studies, this led to inflammation only in the males, explaining why they were so sensitive to the hair-prick test, Sorge and Mogil reported in 2011 (ref. 1). The microglia remained quiet in females, which seemed to account for their indifference to Sorge poking their paws with fine hairs.

To better understand why male and female mice dealt with pain so differently, Sorge and Mogil turned to a pain source that affects all mice. They injured the animals' sciatic nerves, which run from the lower back down each perception to oestrogen, a hormone that controls the development of the uterus, ovaries and breasts, and which regulates the menstrual cycle. Oestrogen can either exacerbate or dull pain, depending on its concentration and location. Testosterone, the hormone involved in development of the penis, testes and prostate, as well as of secondary characteristics such as body hair, has received much less attention from pain researchers, although studies suggest it can reduce pain ${ }^{3}$, and some people with chronic pain take testosterone treatments ${ }^{4}$.

In the case of microglia and pain hypersensitivity, Mogil's research points squarely at testosterone as the control switch for pain pathways. In the 2011 and 2015 studies $^{1,2}$, when Sorge tested castrated male mice, which have low testosterone levels, the animals exhibited a response similar to females. And when the researchers provided testosterone to castrated males, or to females, the pain pathway switched to one dependent on microglia.

Since then, researchers have continued to leg. This led to a form of chronic pain that happens when the body's pain-detecting system is damaged or malfunctioning. It caused both male and female mice to become extra sensitive to touch.

Yet even in this case, there were differences. Microglia seemed to have a prominent role in the pain of males, but not in that of female mice $^{2}$. Sorge and a team of collaborators from three institutions found that, no matter how they blocked microglia, this eliminated the pain hypersensitivity in males alone.

It's not that females were immune to pain. They were just as bothered by nerve injury as the males were, but they weren't using microglia to become hypersensitive to touch. Mogil and Sorge wondered whether another immune component, called a T cell, was behind the chronic pain in females. These cells have a known role in pain sensitization in mice.

Sorge tried the same nerve injury in female mice lacking $\mathrm{T}$ cells. They still became hypersensitive to the fine hairs, but the mechanism now seemed to occur through microglia. In females lacking $\mathrm{T}$ cells, blocking the activity of microglia prevented this pain response, just as it did in males. And when the researchers transferred $\mathrm{T}$ cells back to female mice that were lacking them, the animals stopped using microglia in nerve-injury pain (see 'Two routes to pain').

The team's findings ${ }^{2}$, reported in 2015, had a big influence on the pain field, says Greg Dussor, a neuropharmacologist at the University of Texas at Dallas. The results showed that even though everybody's pain might look similar from the outside, scientists can't assume it's the same on the inside.

\section{PAIN POINTS}

If animals can switch between pain pathways, what controls the switch? Researchers have long attributed sex differences in pain find evidence shoring up the importance of microglia - and the cells' enzymes and receptors - in male mice experiencing pain. And the phenomenon isn't restricted to mice: one of Mogil's collaborators, neuroscientist Michael Salter, also found microglial receptors at work in male rats that had hypersensitivity from nerve injury ${ }^{5}$. Salter, who is chief of research at the Hospital for Sick Children in Toronto, Canada, is now investigating the question in macaques, which are likely to process pain in a more similar way to humans.

It's much harder to investigate these pain pathways in people, but clues are emerging. Neuropharmacologist Ted Price, at the University of Texas at Dallas, and his collaborators have found preliminary evidence, published this month ${ }^{6}$, of differences in how immune cells contribute to pain in people.

They're working with nerve tissue removed from individuals with cancer, whose tumours had invaded their spines. In nerves excised from men experiencing pain, Price's team found signs of inflammation caused by an immune cell called a macrophage. These cells serve a similar function to microglia. In women who were in pain, however, the more important players seemed to be nerve cells themselves and a short stretch of protein building blocks (called a peptide) that stimulates nerve growth. The results suggest parallels between human and rodent sex differences, says Price.

But immune cells and hormones don't fully explain pain differences. For instance, Sarah Linnstaedt, a translational biologist at the University of North Carolina Medical Center in Chapel Hill, has found hints that some women might have a genetic predisposition to chronic pain. Her team has identified a suite of RNA molecules in the bloodstream that are more likely to be elevated in women who develop chronic neck, shoulder or back pain after a 
motor-vehicle accident. Many of these RNA molecules are encoded by genes on the $\mathrm{X}$ chromosome, of which there are two copies in most women ${ }^{7}$.

That's useful information, says Linnstaedt. "It will enable us to develop new therapeutics that can either be used specifically in women, or at higher doses in women."

\section{DRUG DIFFERENTIAL}

Others are thinking about sex-specific pain treatments, too. In a study published online in November 2018, Price and his team reported that a diabetes drug called metformin reduces microglial populations surrounding sensory neurons in the spinal cord. They also showed that the drug blocks pain hypersensitivity from nerve damage only in male mice ${ }^{8}$. "It didn't do anything in the females; in fact, it got a little bit worse," says Price, who has a theory as to why: to enter the nervous system, metformin relies on a protein that's expressed at higher levels in cells from males. Higher doses didn't make a difference in females, however, presumably because the medication was trapped outside the nerves.

Higher doses do help females receiving one of the oldest pain drugs in the pharmacy: morphine. Women and female rodents both usually require higher doses of morphine to achieve the same pain relief as men and male rodents, says Anne Murphy, a neuroscientist at Georgia State University in Atlanta. She's one of a handful of researchers who was studying sex differences well before the NIH changed its guidelines.

Microglia are also behind morphine's differing effects, Murphy's team reported ${ }^{9}$ in 2017. The drug dulls pain by blocking neurons in a brain region called the periaqueductal grey, or PAG. But the drug can also activate microglia there, counteracting morphine's pain-relieving effects. This is exactly what happens in female rats, which have more active microglia in the PAG than males have. When rats were treated with morphine before the scientists applied a hot light beam to their paws, the female animals had more inflammation in the PAG and pulled back their legs more quickly than did males given the same dose. When Murphy's team blocked morphine's effects on microglia, males and females responded to the pain in a similar way'.

There's at least one drug already on the market that scientists have reason to think might work differently across sexes. In 2018, the US Food and Drug Administration approved migraine treatments based on antibodies against CGRP, a peptide found in the nervous system that is involved in these kinds of headache. Migraines affect three times as many women as men.

In an as-yet-unpublished study of mice and rats, a team led by Price and Dussor applied

\section{TWO ROUTES TO PAIN}

Injuries to peripheral nerves - those connecting the brain and the spinal cord to the rest of the body - can cause increased sensitivity to pain. In male mice, this response depends on immune cells in the spinal cord called microglia. In females, it is T cells that seem to control pain.

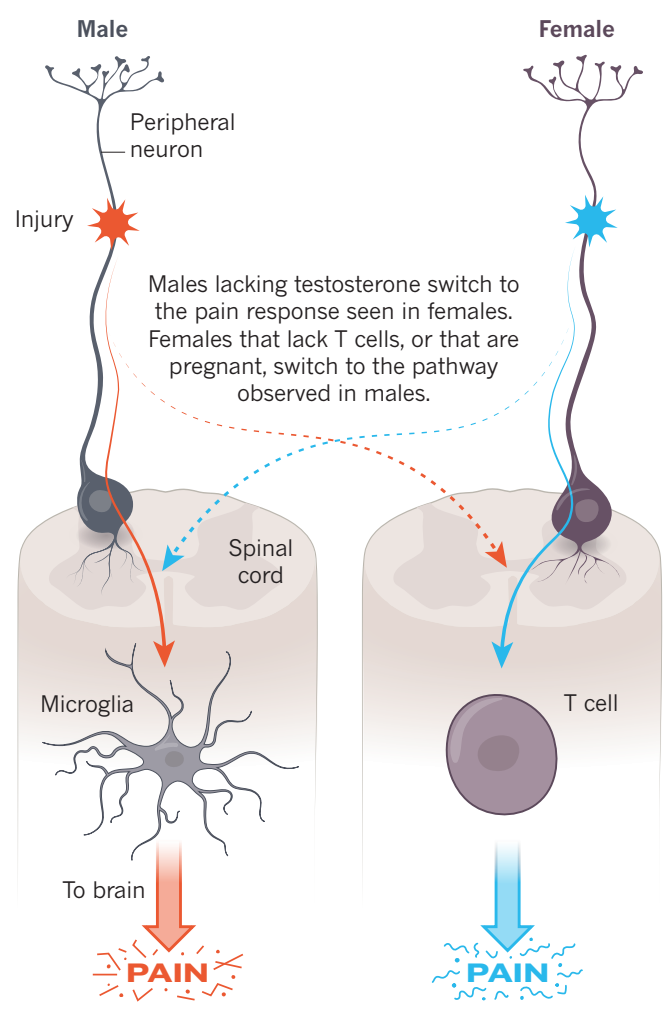

CGRP to the thick membrane surrounding the brain. In females, the peptide created a response that looked like a migraine: the animals grimaced and their faces were hypersensitive to touch. In males: "Nothing," says Dussor. Modern anti-CGRP medicines might work better in women than in men, he adds - but the drug's clinical trials didn't check for such effects.

That's typical of many drug trials. They usually include men and women, but the numbers of each often aren't high enough to suss out differences. There's a real possibility that pain drugs that failed clinical trials in the past might have succeeded if they had been tested separately by sex, says Price. "It seems really obvious," he adds, "but nobody was really doing it."

\section{PERSONALIZED PILLS}

Chessel, at AstraZeneca, would be happy to develop a pain drug that works only in people of a certain sex. But the sex of study participants and animal subjects is driven by practicality, ethical concerns and government regulations, he says. AstraZeneca uses female rodents in most of its preclinical pain research because they're less aggressive and easier to house and handle than males. In early clinical trials, safety is the focus, so companies often exclude people who could become pregnant. As a result, drugs are mostly trialled on men and on women who are past menopause.

Even if scientists develop drugs that are targeted to male- or female-specific pain pathways, these might not be enough. It might be best to customize drugs more closely, to take into account the spectrum of genetics, hormone levels and anatomical development.

Little research has been done on pain mechanisms in people who don't fit into a binary definition of sex and gender. In one study, researchers in Italy surveyed transgender people undergoing hormone treatment. They found that 11 out of 47 people who transitioned from male to female reported pain issues that arose after the transition. Six out of 26 people transitioning from female to male reported that their pain problems lessened after taking testosterone ${ }^{10}$.

On the basis of his team's experiments with castration and testosterone treatments in mice, Mogil thinks that pain pathways will be determined by hormone levels. He predicts that people with more than a certain threshold of testosterone will have pain mechanisms associated with males, and those whose testosterone falls below that level will experience pain through mechanisms common in females.

Pain responses also seem to change throughout life, around the time hormone levels rise or fall. Studies looking only at biological sex have found that, at puberty, the rates of pain conditions rise more in girls than in boys. And as people age, and some hit menopause, hormonal levels change again, and sex differences in chronic pain rates begin to disappear. Pregnancy changes pain responses, too. Mogil's group reported in 2017 that, early in pregnancy, mice switch from a typically female, microglia-independent mechanism of pain sensitization to a more male-associated one that involves microglia. By late pregnancy, the animals don't seem to feel chronic pain at all ${ }^{11}$.

But he's no longer one of a few scientists looking for such sex differences. "People are finding this left, right and centre now," says Mogil. "I don't think we know the half of it at this point."

\section{Amber Dance is a freelance writer in Los Angeles, California.}

1. Sorge, R. E. et al. J. Neurosci. 31, 15450-15454 (2011).

2. Sorge, R. E. et al. Nature Neurosci. 18, 1081-1083 (2015).

3. Nag, S. \& Mokha, S. S. Neurosci. Lett. 467, 48-52 (2009).

4. White, H. D. \& Robinson, T. D. Int. Immunopharmacol. 27, 244-248 (2015).

5. Mapplebeck, J. C. S. et al. Pain 159, 1752-1763 (2018).

6. North, R. Y et al. Brain https://doi.org/10.1093/ brain/awz063 (2019)

7. Yu, S. et al. Am. J. Med. Genet. B https://doi. org/10.1002/ajmg.b.32706 (2018).

8. Inyang, K. E. et al. Pharmacol. Res. 139, 1-16 (2019).

9. Doyle, H. H., Eidson, L. N., Sinkiewicz, D. M. \& Murphy, A. Z. J. Neurosci. 37, 3202-3214 (2017)

10.Aloisi, A. M. et al. Pain 132, S60-S67 (2007).

11. Rosen, S. F. et al. J. Neurosci. 37, 9819-9827 (2017). 